\title{
Error-detecting properties of languages is
}

\author{
Stavros Konstantinidis *, Amber O'Hearn \\ Department of Mathematics and Computing Science, Saint Mary's University, Halifax, NS, \\ Canada $\mathrm{B} 3 \mathrm{H} 3 \mathrm{C} 3$
}

Received June 2000; revised April 2001; accepted May 2001

Communicated by A. Salomaa

\begin{abstract}
The language property of error-detection ensures that the communications medium cannot transform a word of the language to another word of the language. In this paper we provide some insights on the notion of error-detection from a language theoretic point of view. We define certain error-detecting properties of languages and codes including the notion of error-detection with finite delay which is a natural extension of unique decodability with finite delay. We obtain results about the error-detecting capabilities of regular and other languages, and of known classes of codes. Moreover, we consider the problem of estimating the optimal redundancy of infinite languages with the property of detecting errors of the deletion type. (c) 2002 Elsevier Science B.V. All rights reserved.
\end{abstract}

Keywords: Channel; Code; Language; Error-detection; Finite delay; Redundancy

\section{Introduction}

Communication of information presupposes the existence of a communications medium and a language of communications which consists of all the possible messages (words) that can be communicated. Normally, the medium, say $\gamma$, is capable of introducing errors in the words of the language, say $L$, and there is the possibility that, when a word $w$ of $L$ is communicated, $\gamma$ returns a word $w^{\prime}$ which is not in $L$ or it is in $L$ but $w^{\prime} \neq w$. These considerations are important, for instance, in the transmission of digital information and in the typesetting of ASCII characters. In the former case, the channel (medium) is capable of substituting symbols of the message with

\footnotetext{
This work was supported by research Grant OGP220259 of the Natural Sciences and Engineering Research Council of Canada.

* Corresponding author.

E-mail addresses: s.konstantinidis@stmarys.ca (S. Konstantinidis), a_ohearn@cs.stmarys.ca (A. O’Hearn).
} 
other symbols and possibly inserting new or deleting existing symbols in the message. Moreover, in this case, the language of communications is usually coded; that is, $L$ is freely generated by a code. In the latter case, the typesetter can be thought of as being the channel that transmits the words of a language to the computer. The communications language in this case is usually not coded, as it could be a natural language or a programming language.

In the above scenarios, the language property of error-detection is of particular interest. Specifically, if the language $L$ is error-detecting for the channel $\gamma$, then $\gamma$ cannot transform a word in $L$ to another word in $L$. As a consequence, when the channel returns a word $w$ which is in $L$ then $w$ is correct. On the other hand, if the returned word is not in $L$, one can be sure that it is not equal to the intended word and then take appropriate action - for example, request that the word be retransmitted. The objective of this paper is to provide some insights on the concept of error-detection by defining certain error-detecting properties of languages, including coded languages, and obtaining some basic results concerning error-detecting capabilities of regular and other languages, and of known classes of codes. To keep the basic definitions general, we use the framework of $P$-channels (see [9]) restricted to the case of finite words. This channel model is very general and includes the case of SID-channels which were presented in [8] and further extended in [10]. SID-channels are discrete channels represented by formal expressions that describe the type of errors permitted and the frequency of those errors. The basic error types are:

$\sigma$ : substitution. It means that a symbol in a message can be replaced with another symbol (of the alphabet $X$ ).

$l$ : insertion. It means that a symbol (of the alphabet $X$ ) can be inserted in a message.

$\delta$ : deletion. It means that a symbol in a message can be deleted, i.e., replaced with the empty word.

We note that, in the context of data communications, errors of types $\imath$ and $\delta$ are called synchronization errors, as they cause, or are caused by, loss of synchronization. The operation $\odot$ is used to combine error types. In particular, we consider the following set of error types

$$
\{\sigma, l, \delta, \sigma \odot \delta, \sigma \odot \imath, \imath \odot \delta, \sigma \odot \imath \odot \delta\}
$$

For every error type $\tau$ the expression $\tau(m, \ell)$ denotes the channel that permits a total of at most $m$ errors of type $\tau$ in any $\ell$ (or less) consecutive symbols of a message-see the next section for a formal definition. In this case, we assume that $m$ and $\ell$ are positive integers.

The paper is organized as follows. The next section gives some basic concepts about words, word factorizations, $P$-channels, and SID-channels. Section 3 defines the basic error-detecting properties of languages, provides examples to illustrate these properties, and contains a few results on error-detection for $P$ - and SID-channels. For example, it is shown that the number of synchronization errors that a regular language can detect is bounded by the cardinality of its syntactic monoid. In Section 4 the concept of 
error-detection with finite delay is introduced which is a natural generalization of the code property of unique decodability with finite delay. It is shown that no coded language is error-detecting with delay 0 for any SID-channel that permits insertions. Section 5 discusses certain error-detecting capabilities of uniform, solid and shuffle codes, and provides a construction of a language which is maximal error-detecting for the channel $\delta(m, \ell)$ and whose redundancy is asymptotically optimal. Finally, Section 6 contains a few concluding remarks.

\section{Basic notation and background}

For a set $S$, the notation $|S|$ represents the cardinality of $S$. The set of positive integers is denoted by $\mathbb{N}$ and $\mathbb{N}_{0}=\mathbb{N} \cup\{0\}$. An alphabet, $X$, is a non-empty set of symbols. A word (over $X$ ) is a mapping $w:\{0,1, \ldots, n-1\} \rightarrow X$ for some $n \in \mathbb{N}_{0}$. In this case, we write $I_{w}$ to denote the domain $\{0,1, \ldots, n-1\}$ of the word $w$. Moreover, as usual, we can denote $w$ by juxtaposing its elements: $w=w(0) w(1) \cdots w(n-1)$. The empty word, $\lambda$, is the unique word with $I_{\lambda}=\emptyset$. The length, $|w|$, of a word $w$ is the number $\left|I_{w}\right|$. The set of all words over $X$ is denoted by $X^{*}$ and $X^{+}=X^{*} \backslash\{\lambda\}$. A language is a subset of $X^{*}$. We write minlen $L$ to denote the length of a shortest word in the language $L$. On the other hand, if $L$ is finite we write maxlen $L$ to denote the length of a longest word in $L$. If all the words in $L$ are of the same length, we say that $L$ is a uniform code. In this case, we use the symbol len $L$ to denote the length of the words in $L$. In the sequel, we use the symbol $X$ for an arbitrary but fixed finite alphabet.

Let $L$ be a language. A factorization over $L$ is a mapping $\varphi:\{0,1, \ldots, n-1\} \rightarrow L$ for some $n \in \mathbb{N}_{0}$. As before, we write $I_{\varphi}$ to indicate the domain $\{0,1, \ldots, n-1\}$ of $\varphi$, and $|\varphi|$ to denote the length of the factorization $\varphi$ which is equal to $\left|I_{\varphi}\right|$. For a factorization $\varphi$ over $L$, we write $[\varphi]$ to denote the word $\varphi(0) \varphi(1) \cdots \varphi(n-1)$, where $n=|\varphi|$. If $|\varphi|=0$ then $[\varphi]=\lambda$. For $n \in \mathbb{N}_{0}$ and $w \in X^{*}$, the symbol $w^{n}$ denotes the word $[\varphi]$ such that $|\varphi|=n$ and $\varphi(i)=w$ for all $i \in I_{\varphi}$. Also, for $W \subseteq X^{*}, W^{n}=\left\{w^{n} \mid w \in W\right\}$ and $W^{\leqslant n}=\bigcup_{i=0}^{n} W^{i}$.

A code (over $X)$ is a non-empty subset $K$ of $X^{+}$such that $[\varphi]=[\psi]$ implies $\varphi=\psi$ for all factorizations $\varphi$ and $\psi$ over $K$. A message over $K$ is a word [ $\varphi]$, where $\varphi$ is a factorization over $K$. Then, $K^{*}$ is the set of all messages over $K$ and $K^{+}$is the set of all non-empty messages. Since a word over $X$ can be viewed as a factorization over $X$, we also refer to words as messages (over $X$ ).

A channel, $\gamma$, is a binary relation over $X^{*}$, namely $\gamma \subseteq X^{*} \times X^{*}$. For the elements of a channel $\gamma$, we prefer to write $\left(y^{\prime} \mid y\right)$ rather than $\left(y^{\prime}, y\right)$. Then, $\left(y^{\prime} \mid y\right) \in \gamma$ means that the word $y^{\prime}$ can be obtained from $y$ through the channel $\gamma$. For a word $y$, we define $\langle y\rangle_{\gamma}$ to be the set of all possible outputs of $\gamma$ when $y$ is used as input; that is,

$$
\langle y\rangle_{\gamma}=\left\{y^{\prime} \in X^{*} \mid\left(y^{\prime} \mid y\right) \in \gamma\right\} .
$$

More generally, for a set of words $Y$, we have $\langle Y\rangle_{\gamma}=\bigcup_{y \in Y}\langle y\rangle_{\gamma}$. 
Definition 1. Let $\gamma$ be a channel, let $Y$ be a subset of $X^{*}$, and let $\varphi$ be a factorization over $Y$. A factorization $\varphi^{\prime}$ over $\langle Y\rangle_{\gamma}$ is $\gamma$-admissible for $\varphi$ if

$$
I_{\varphi^{\prime}}=I_{\varphi} \quad \text { and } \quad \varphi^{\prime}(i) \cdots \varphi^{\prime}(i+k) \in\langle\varphi(i) \cdots \varphi(i+k)\rangle_{\gamma},
$$

for all $i \in I_{\varphi}$ and $k \in \mathbb{N}_{0}$ with $i+k \in I_{\varphi}$.

Example 1. Consider the word $y=a a b b a a$, where $a, b \in X$, and its factorization $\varphi$ over $K=\{a a, b b\}$ such that $\varphi=(a a, b b, a a)$. Consider also a channel $\gamma$ that allows at most one deletion in any 2 consecutive input symbols - as we shall see next, $\gamma$ is an SIDchannel. Then, $y^{\prime}=a b a a$ is a possible output in $\langle y\rangle_{\gamma}$ if one deletes the symbols $y(0)$ and $y(2)$ in $y$. Then, the factorization $\varphi^{\prime}$ of $y^{\prime}$ over $\langle K\rangle_{\gamma}$ such that $\varphi^{\prime}=(a, b, a a)$ is $\gamma$-admissible for $\varphi$. On the other hand, for the same channel $\gamma$, and for $K=\{a b, b a\}$ and $y=a b b a$, one has the following: $\varphi=(a b, b a)$ is a factorization of $y$ over $K$ and $\varphi^{\prime}=(a, a)$ is a factorization of $y^{\prime}=a a$ over $\langle K\rangle_{\gamma}$ such that $\varphi^{\prime}(i) \in\langle\varphi(i)\rangle_{\gamma}$ for $i \in\{0,1\}$. But $y^{\prime} \notin\langle\varphi(0) \varphi(1)\rangle_{\gamma}$ since the symbols $y(1)$ and $y(2)$ of $y$ cannot be both deleted. Hence, $\varphi^{\prime}$ is not $\gamma$-admissible for $\varphi$.

In the sequel, we consider only channels $\gamma$ satisfying the following natural conditions: $\left(\mathscr{P}_{1}\right)$ Input factorizations arrive as $\gamma$-admissible output factorizations: If $\left(y^{\prime} \mid y\right) \in \gamma$ and $\varphi$ is a non-empty factorization of $y$ over some subset $Y$ of $X^{*}$, then there is a factorization $\varphi^{\prime}$ of $y^{\prime}$ over $\langle Y\rangle_{\gamma}$ which is $\gamma$-admissible for $\varphi$.

$\left(\mathscr{P}_{2}\right)$ Error-free messages can be received independently of the context: If $\left(y^{\prime} \mid y\right) \in \gamma$ then $\left(x y^{\prime} z \mid x y z\right) \in \gamma$, for all $x, z \in X^{*}$.

$\left(\mathscr{P}_{3}\right)$ Empty input can result into empty output: $(\lambda \mid \lambda) \in \gamma$.

Channels satisfying properties $\mathscr{P}_{1}-\mathscr{P}_{3}$ are called $P_{*}$-channels. They differ from the $P$-channels defined in [9] only in the finiteness type of the inputs and outputs; that is, $P_{*}$-channels allow only finite words to be used as opposed to $P$-channels. Consequently, property $\mathscr{P}_{0}$ of $P$-channels is omitted here. We note that properties $\mathscr{P}_{2}$ and $\mathscr{P}_{3}$ imply $(y \mid y) \in \gamma$ for all $y \in X^{*}$.

We now define a certain class of SID-channels - see [10] for the full class of SIDchannels. Although the formal definition is not required for the proofs in the present paper, it is included here so that the reader can clearly understand how these channels affect messages. The main tool in the definition is the set of error functions. An error function is represented by a string of basic error functions and is applied on messages on a symbol-by-symbol basis. Consider, for instance, the message $x=a b a b$ over the alphabet $\{a, b\}$ and consider a channel that would allow one substitution, one insertion and one deletion in $x$. As $x=\lambda a \lambda b \lambda a \lambda b \lambda$, we see that there are four possible positions for a substitution (the four symbols of $x$ ), five possible positions for an insertion (the five $\lambda \mathrm{s}$ ), and four possible positions for a deletion. Thus, baaa is a possible output from $x$ by inserting a $b$ in front of $x$, substituting the first $b$ of $x$ with $a$, and deleting the last $b$ of $x$. This effect can be expressed by applying the sequence of basic error functions $\mathbf{i}_{b}, \mathbf{e}, \mathbf{e}, \mathbf{s}, \mathbf{e}, \mathbf{e}, \mathbf{e}, \mathbf{d}, \mathbf{e}$ to each of the nine positions of $x$, respectively, where $\mathbf{e}$ 
is the identity function (no error at that position), $\mathbf{i}_{b}$ is a function that replaces $\lambda$ by $b$ (insertion in that position), $\mathbf{s}$ is the function that reverses symbols, and $\mathbf{d}$ is a function that replaces an alphabet symbol by $\lambda$ (deletion at that position). Thus, if we consider the error function $\mathbf{h}=\mathbf{i}_{b}$ eeseeede, then

$$
\mathbf{h}(x)=\mathbf{i}_{b}(\lambda) \mathbf{e}(a) \mathbf{e}(\lambda) \mathbf{s}(b) \mathbf{e}(\lambda) \mathbf{e}(a) \mathbf{e}(\lambda) \mathbf{d}(b) \mathbf{e}(\lambda)=\text { baaa } .
$$

Generally, when $x$ is a message of length $n$, an error function $\mathbf{h}$ can be applied on $x$ provided that $|\mathbf{h}|=2 n+1$.

The alphabet $G$ of basic error functions consists of the following symbols: The symbol $\mathbf{d}$ which denotes the deletion function $\mathbf{d}: X \rightarrow\{\lambda\}$ such that $\mathbf{d}(a)=\lambda$ for all $a \in X$. For each non-empty word $u$, the symbol $\mathbf{i}_{u}$ which denotes the insertion function $\mathbf{i}_{u}:\{\lambda\} \rightarrow\{u\}$. Symbols of the form $\mathbf{s}$ which denotes a substitution function $\mathbf{s}: X \rightarrow X$ such that $\mathbf{s}(a) \neq a$, for all $a \in X$. Finally, the symbol $\mathbf{e}$ which denotes the identity function $\mathbf{e}: X \cup\{\lambda\} \rightarrow X \cup\{\lambda\}$ such that $\mathbf{e}(a)=a$, for all $a \in X \cup\{\lambda\}$.

We set $G_{\varepsilon}=\{\mathbf{e}\}, G_{\delta}=\{\mathbf{d}\}, G_{l}=\left\{\mathbf{i}_{u} \mid u \in X^{+}\right\}$, and $G_{\sigma}$ equal to the set of all substitution functions $\mathbf{s}$ from $X$ into $X$. Hence, the alphabet of error function symbols can be written as $G=G_{\varepsilon} \cup G_{\sigma} \cup G_{l} \cup G_{\delta}$. Moreover, for every error type $\tau$, the set $G_{\tau}$ consists of the basic error functions of all the error types that occur in $\tau$. For example, $G_{\sigma \odot \iota}=G_{\sigma} \cup G_{l}$ and $G_{\sigma \odot \iota \odot \delta}=G_{\sigma} \cup G_{l} \cup G_{\delta}$.

Definition 2. An error function is a word $\mathbf{h}$ over $G$ such that $|\mathbf{h}|$ is odd and, for all $i \in I_{\mathbf{h}}$,

$$
\mathbf{h}(i) \in \begin{cases}G_{\varepsilon} \cup G_{l} & \text { if } i \text { is even; } \\ G_{\varepsilon} \cup G_{\delta} \cup G_{\sigma} & \text { if } i \text { is odd. }\end{cases}
$$

We use the symbol $\mathscr{H}$ to denote the set of error functions. Moreover, if $\tau$ is an error type, we write $\mathscr{H}_{\tau}$ for the set $\mathscr{H} \cap\left(G_{\tau} \cup G_{\varepsilon}\right)^{*}$.

The set of error functions is equipped with a product operation, '.', such that $(\mathscr{H}, \cdot)$ is a monoid whose neutral element is e. Specifically, if $\mathbf{h}$ and $\mathbf{g}$ are error functions such that $|\mathbf{h}|=2 n+1$, their product $\mathbf{h} \cdot \mathbf{g}$ is defined as the usual concatenation of words, except at the point where the last symbol of $\mathbf{h}$ and the first symbol of $\mathbf{g}$ are concatenated; that is the symbols $\mathbf{h}(2 n)$ and $\mathbf{g}(0)$ become one symbol, $\mathbf{c}$, as follows:

$$
\mathbf{c}= \begin{cases}\mathbf{h}(2 n) & \text { if } \mathbf{g}(0)=\mathbf{e} \\ \mathbf{g}(0) & \text { if } \mathbf{h}(2 n)=\mathbf{e} \\ \mathbf{i}_{u_{1} u_{2}} & \text { if } \mathbf{h}(2 n)=\mathbf{i}_{u_{1}} \text { and } \mathbf{g}(0)=\mathbf{i}_{u_{2}}\end{cases}
$$

For example, $($ ede $) \cdot\left(\mathbf{i}_{a} \mathbf{s e}\right)=\mathbf{e d i}_{a} \mathbf{s e},($ ede $) \cdot($ ese $)=$ edese, and $\left(\right.$ edi $\left._{a}\right) \cdot\left(\mathbf{i}_{b} \mathbf{s e}\right)=\mathbf{e d i}_{a b} \mathbf{s e}$ Finally we note that when $\mathbf{h}$ can be written as $\mathbf{f}_{1} \cdot \mathbf{g} \cdot \mathbf{f}_{2}$, the error function $\mathbf{g}$ is called an $\mathscr{H}$-factor of $\mathbf{h}$. 
The function $\mathscr{N}: \mathscr{H} \rightarrow \mathbb{N}_{0}$ is defined such that, for every $\mathbf{h} \in \mathscr{H}, \mathscr{N}(\mathbf{h})$ is the number of errors that occur in $\mathbf{h}$. More formally, $\mathscr{N}(\mathbf{h})$ is the sum of

- the number of symbols $\mathbf{d}$ and $\mathbf{s}$ that occur in $\mathbf{h}$, plus

- $|u|$ for each symbol $\mathbf{i}_{u}$ that occurs in $\mathbf{h}$.

For example, $\mathscr{N}($ eeede $)=1$ and $\mathscr{N}\left(\right.$ eei $_{a}$ eedeseei $\left._{a b}\right)=5$.

Now for every positive integers $m$ and $\ell$ and for every error type $\tau$, the set $\mathscr{H}_{m, \ell}(\tau)$ consists of all the error functions $\mathbf{h}$ in $\mathscr{H}_{\tau}$ such that $\mathscr{N}(\mathbf{g}) \leqslant m$ for every $\mathscr{H}$-factor $\mathbf{g}$ of $\mathbf{h}$ with $|\mathbf{g}| \leqslant 2 \ell+1$. Finally, the expression $\tau(m, \ell)$ denotes the SID-channel:

$$
\left\{\left(y^{\prime} \mid y\right) \mid y \in X^{*} \quad \text { and } \quad \exists \mathbf{h}, y^{\prime}=\mathbf{h}(y), \mathbf{h} \in \mathscr{H}_{m, \ell}(\tau)\right\} .
$$

That is, $\tau(m, \ell)$ is the channel in which an input message $y$ can result in the output message $y^{\prime}$ using at most $m$ errors of type $\tau$ in every $\ell$ consecutive symbols of $y$. It can be shown that every SID-channel is a $P_{*}$-channel [9].

Example 2. Consider the word $x=$ aaaaaaa and the SID-channel $\gamma=\imath \odot \delta(2,5)$ that permits at most 2 insertions and deletions in any 5 consecutive symbols. Let $y=$ abaaaaab and let $z=a b b a a a a a b a$. Observe that $y$ can be obtained from $x$ when $\gamma$ deletes $x(2)$, inserts a $b$ between $x(0)$ and $x(1)$, and inserts a $b$ at the end of $x$; that is, $y=\mathbf{h}(x)$ where $\mathbf{h}=$ eei $_{b}$ eedeeeeeeei $_{b}$. Hence, as $\mathbf{h} \in \mathscr{H}_{2,5}(l \odot \delta), y \in\langle x\rangle_{\gamma}$. On the other hand, to obtain $z$ from $x$ using a minimum number of errors, one has to insert three $b$ 's in the segment $x(1) \cdots x(5)$-including the endpoints of that segment - of length 5; that is, $z=\mathbf{h}(x)$ where $\mathbf{h}=\mathbf{e e i}_{b b}$ eeeeeeeei $_{b}$ ee. Then, for the $\mathscr{H}$-factor $\mathbf{g}=\mathbf{i}_{b b}$ eeeeeeeei $_{b}$ of h one has $|\mathbf{g}|=11=2 \cdot 5+1$ but $\mathscr{N}(\mathbf{g})=3$. Hence, $z \notin\langle x\rangle_{\gamma}$.

\section{Error detection: definitions and basic results}

The classical theory of error-correcting codes deals with channels that permit only substitution errors and considers primarily uniform codes. In that context, a uniform code $K$ is said to be $m$-error-detecting if $v_{1} \in\left\langle v_{2}\right\rangle_{\gamma}$ implies $v_{1}=v_{2}$, for all codewords $v_{1}$ and $v_{2}$, where $\gamma=\sigma(m, \ell)$ and $\ell$ is the length of the words in $K$-see [5] or [16]. In this section, we extend the notion of error-detection to the case of arbitrary channels and codes. Moreover, we also define error-detection for arbitrary languages motivated by the fact that natural languages are not coded but they possess certain error-detecting capabilities (see [1]).

A language $L$ is called coded if there is a code $C$ such that $L=C^{*}$. If $C_{1}$ and $C_{2}$ are codes satisfying $C_{1}^{*}=C_{2}^{*}$ then $C_{1}=C_{2}$ (see [18]). Hence, if $L$ is a coded language there is a unique code $C$ such that $L=C^{*}$. We write $C_{L}$ to denote that code.

Definition 3. Let $\gamma$ be a channel.

(i) A language $L$ is error-detecting for $\gamma$, if

$$
\forall w_{1}, w_{2} \in L \cup\{\lambda\}, \quad w_{1} \in\left\langle w_{2}\right\rangle_{\gamma} \rightarrow w_{1}=w_{2} .
$$

(ii) A code $K$ is $(\gamma, *)$-detecting, if the coded language $K^{*}$ is error-detecting for $\gamma$. 
The concepts of $(\gamma, *)$-detecting code and $(\gamma, *)$-correcting code are defined in [9]a code $K$ is $(\gamma, *)$-correcting if $\left\langle w_{1}\right\rangle_{\gamma} \cap\left\langle w_{2}\right\rangle_{\gamma} \neq \emptyset$ implies $w_{1}=w_{2}$, for all $w_{1}, w_{2} \in K^{*}$. For the first part of the above definition we note that the use of " $w_{1}, w_{2} \in L \cup\{\lambda\}$ " as opposed to " $w_{1}, w_{2} \in L$ " is justified as follows. First, it should not be possible for the channel $\gamma$ to return a non-empty word in $L$ when nothing is sent to $\gamma$, i.e., when the input used is $\lambda$. That is, $w_{1} \in\langle\lambda\rangle_{\gamma}$ and $w_{1} \in L \cup\{\lambda\}$ implies $w_{1}=\lambda$. Similarly, the channel should not be capable of erasing completely a non-empty word of $L$. That is, $\lambda \in\left\langle w_{2}\right\rangle_{\gamma}$ and $w_{2} \in L \cup\{\lambda\}$ implies $w_{2}=\lambda$. These observations do not eliminate from consideration channels that insert or delete symbols. Instead, they ensure that when an error-detecting language is used for $\gamma$, it is impossible that $\gamma$ can erase or introduce an entire non-empty word of $L$.

Example 3. Every uniform code $K$ is error-detecting for the channel $\gamma=\imath(m, \ell)$, provided len $K>m$. Indeed, as only insertions are permitted, $x \in\langle v\rangle_{\gamma}$ implies $|v| \leqslant|x|$; therefore, $\lambda \in\langle v\rangle_{\gamma}$ and $v \in K \cup\{\lambda\}$ imply $v=\lambda$. On the other hand, as $m<\operatorname{len} K$, one has that $v \in\langle\lambda\rangle_{\gamma}$ and $v \in K \cup\{\lambda\}$ imply $v=\lambda$. Now let $v_{1}$ and $v_{2}$ be codewords of $K$ such that $v_{1} \in\left\langle v_{2}\right\rangle_{\gamma}$. Then, $\left|v_{1}\right| \geqslant\left|v_{2}\right|$. In particular, $\left|v_{1}\right|=\left|v_{2}\right|$ if and only if no insertion occurs in $v_{2}$, if and only if $v_{1}=v_{2}$. Hence, as $K$ is uniform, $v_{1}=v_{2}$. Analogously, one can verify that every uniform code $K$ is error detecting for $\delta(m, \ell)$, provided len $K>m$.

Example 4. One can verify that the code $K_{0}=\{a a a, b b b\}$, where $a, b \in X$, is errordetecting for the channel $\gamma=\sigma \odot \imath \odot \delta(1,3)$. But $K_{0}$ is not $(\gamma, *)$-detecting. Indeed, consider the messages $w_{2}=(a a a)^{3}$ and $w_{1}=(a a a)^{2}$ such that $w_{1} \neq w_{2}$. Then, $w_{1} \in\left\langle w_{2}\right\rangle_{\gamma}$ by deleting appropriately three symbols from $w_{2}$.

Example 5. Consider the code $K_{1}=\left\{v_{1}, v_{2} \mid v_{1}=a a b b b, v_{2}=a b a b a b b\right\}$, where $a, b \in$ $X$, and the channel $\gamma=\delta(1,7)$. Then, $\left\langle v_{1}\right\rangle_{\gamma}=\left\{v_{1}, a b b b, a a b b\right\}$ and

$$
\left\langle v_{2}\right\rangle_{\gamma}=\left\{v_{2}, \text { bababb, aababb, abbabb, abaabb, ababbb, ababab }\right\} .
$$

Obviously, the empty word cannot be obtained from either $v_{1}$ and $v_{2}$ through $\gamma$ and, conversely, neither of $v_{1}$ and $v_{2}$ can be obtained from the empty word through $\gamma$. Moreover, $v_{1} \notin\left\langle v_{2}\right\rangle_{\gamma}$ and $v_{2} \notin\left\langle v_{1}\right\rangle_{\gamma}$. Hence, $K_{1}$ is error-detecting for $\gamma$. Now we claim that $K_{1}$ is $(\gamma, *)$-detecting. Indeed, note first that $\lambda \notin\langle w\rangle_{\gamma}$ and $w \notin\langle\lambda\rangle_{\gamma}$ for all $w \in K_{1}^{+}$. Now consider two messages $w_{1}$ and $w_{2}$ in $K_{1}^{+}$such that $w_{1} \in\left\langle w_{2}\right\rangle_{\gamma}$. Then, $w_{1}=\left[\kappa_{1}\right]$ and $w_{2}=\left[\kappa_{2}\right]$ for some factorizations $\kappa_{1}$ and $\kappa_{2}$ over $K_{1}$. By property $\mathscr{P}_{1}$ of the channel $\gamma$, there is a factorization $\psi$ which is $\gamma$-admissible for $\kappa_{2}$ such that $[\psi]=w_{1}=\left[\kappa_{1}\right]$ and $\psi(i) \in\left\langle\kappa_{2}(i)\right\rangle_{\gamma}$ for all $i \in I_{\psi}=I_{\kappa_{2}}$. It is sufficient to show that $\psi=\kappa_{1}$; then, as $K_{1}$ is error-detecting for $\gamma, \kappa_{1}(i) \in\left\langle\kappa_{2}(i)\right\rangle_{\gamma}$ implies $\kappa_{1}(i)=\kappa_{2}(i)$ for all $i$ in $I_{\kappa_{2}}$. So consider the word $\kappa_{1}(0)$ of $K_{1}$ which is a prefix of both, [ $\kappa_{1}$ ] and [ $\psi$ ]. If $\kappa_{1}(0)=v_{1}$ then $\psi(0)=v_{1}$ or $\psi(0)=a a b b$. The second case implies $\psi(1)=b a b a b b$ which is impossible, as two deletions would occur in $\kappa_{2}(0) \kappa_{2}(1)$ within a segment of length less than 7 . Hence, $\psi(0)=v_{1}$ as well. Similarly, one verifies that if $\kappa_{1}(0)=v_{2}$ 
then $\psi(0)=v_{2}$ as well. Hence, $\psi(0)=\kappa_{1}(0)$ and $\psi(1) \psi(2) \cdots=\kappa_{1}(1) \kappa_{1}(2) \cdots$. The same argument can be applied repeatedly to obtain $\psi(i)=\kappa_{1}(i)$ for all $i$ in $I_{\psi}$.

In many cases, the code property of $(\gamma, *)$-detection can be studied in terms of the weaker notion of $(\gamma, t)$-detecting code, where $t \in \mathbb{N}_{0}$.

Definition 4. Let $\gamma$ be a channel and let $t \in \mathbb{N}_{0}$. A code $K$ is $(\gamma, t)$-detecting, if

$$
\forall w_{1} \in K^{\leqslant t} \forall w_{2} \in K^{*}, \quad w_{1} \in\left\langle w_{2}\right\rangle_{\gamma} \rightarrow w_{1}=w_{2} .
$$

The following proposition describes certain relationships between the error-detecting properties given in Definitions 3 and 4.

Proposition 1. For every $P_{*}$-channel $\gamma$, the following statements hold true:

(i) For all $t \in \mathbb{N}_{0}$, if a code $K$ is $(\gamma, t+1)$-detecting then $K$ is $(\gamma, t)$-detecting and $K$ is error-detecting for $\gamma$.

(ii) $A$ code $K$ is $(\gamma, *)$-detecting if and only if $K$ is $(\gamma, t)$-detecting for all $t \in \mathbb{N}_{0}$.

(iii) For all $t \in \mathbb{N}_{0}$, there exists an SID-channel $\gamma$ and a code $K$ such that $K$ is $(\gamma, t)$-detecting but $K$ is not $(\gamma, t+1)$-detecting.

Proof. (i) Consider a code $K$ which is $(\gamma, t+1)$-detecting and the messages $w_{1} \in K^{\leqslant t}$ and $w_{2} \in K^{*}$ such that $w_{1} \in\left\langle w_{2}\right\rangle_{\gamma}$. Let $v \in K$. By property $\mathscr{P}_{2}$ of the channel $\gamma$, one has $w_{1} v \in\left\langle w_{2} v\right\rangle_{\gamma}$. As $w_{1} v \in K^{\leqslant t+1}$ and $w_{2} v \in K^{*}$, it follows that $w_{1} v=w_{2} v$. Hence, $w_{1}=w_{2}$ and $K$ is $(\gamma, t)$-detecting. Moreover, it is easy to see that $K$ is error-detecting for $\gamma$.

(ii) The statement follows easily from the definitions.

(iii) For each $t$ in $\mathbb{N}_{0}$ consider the SID-channel $\gamma=\gamma(t)=\delta(1, t+2)$ and the code $K=K(t)=\left\{a^{t+2}\right\}$, where $a \in X$. First we show that $K$ is $(\gamma, t)$-detecting and then that $K$ is not $(\gamma, t+1)$-detecting.

Let $w_{1} \in K^{m}$ and $w_{2} \in K^{n}$ such that $w_{1} \in\left\langle w_{2}\right\rangle_{\gamma}$ and $m, n \in \mathbb{N}_{0}$ with $m \leqslant t$. As only deletions are permitted, $\left|w_{1}\right| \leqslant\left|w_{2}\right|$. If $\left|w_{1}\right|=\left|w_{2}\right|$ then $w_{1}=w_{2}$ as required. On the other hand, we show that the assumption $\left|w_{1}\right|<\left|w_{2}\right|$ leads to a contradiction. Indeed, as $|K|=1$, this assumption implies $m+1 \leqslant n$. Now as $w_{2}$ consists of $n$ codewords each of length $t+2$, at most one symbol can be deleted in each codeword and, therefore, at most $n$ deletions can occur in $w_{2}$. Hence, $\left|w_{1}\right| \geqslant\left|w_{2}\right|-n$ which together with $m+1 \leqslant n$ imply

$$
m(t+2) \geqslant n(t+2)-n \Rightarrow n \leqslant \frac{m(t+2)}{t+1} \Rightarrow m+1 \leqslant \frac{m(t+2)}{t+1} \Rightarrow t+1 \leqslant m .
$$

The last inequality, however, contradicts $m \leqslant t$.

Now we show that $K$ is not $(\gamma, t+1)$-detecting. Let $w_{1}=\left(a^{t+2}\right)^{t+1} \in K^{\leqslant t+1}$ and $w_{2}=\left(a^{t+2}\right)^{t+2} \in K^{*}$. Clearly $w_{1} \neq w_{2}$. On the other hand, one has that $w_{1} \in\left\langle w_{2}\right\rangle_{\gamma}$ by deleting appropriately one $a$ in every $t+2$ consecutive symbols of $w_{2}$.

The following result poses a certain restriction on the words of $(\gamma, *)$-detecting codes for SID-channels that involve insertions or deletions, and gives a certain bound on the 
number of insertion/deletion errors that a regular language can detect. The symbol syn $L$ denotes the syntactic monoid of the language $L$. It is well-known that a language $L$ is regular if and only if syn $L$ is finite (see [17]).

Theorem 1. Let $m, \ell \in \mathbb{N}$, let $\tau$ be an error type that contains the deletion or insertion type, and let $\gamma=\tau(m, \ell)$. Then, the following statements hold true:

(i) If a code $K$ is $(\gamma, *)$-detecting then $x^{n} \notin K$ for all $x \in X^{\leqslant m}$ and for all $n \in \mathbb{N}$.

(ii) If a regular language $L$, other than $\emptyset$ and $\{\lambda\}$, is error-detecting for $\gamma$ then $m<|\operatorname{syn} L|$.

Proof. (i) Assume $\tau$ contains $\delta$ and suppose there are $n \in \mathbb{N}$ and $x \in X^{\leqslant m} \cap X^{+}$such that $x^{n} \in K$. Then, $x^{n(n-1) \ell}$ and $x^{n(n \ell)}$ are different messages in $K^{*}$. As $\left|x^{n \ell}\right| \geqslant \ell$, the channel can delete $x$ in each of the $n$ factors $x^{n \ell}$ of $x^{n(n \ell)}$. Hence, $x^{n(n-1) \ell} \in\left\langle x^{n(n \ell)}\right\rangle_{\gamma}$ and, therefore, $K$ is not $(\gamma, *)$-detecting. The case of $\tau$ containing $l$ is similar.

(ii) Assume $L$ is regular, with $L \notin\{\emptyset,\{\lambda\}\}$, and suppose $m \geqslant \mid$ syn $L \mid$. Let $w \in L \backslash\{\lambda\}$. If $|w|<|\operatorname{syn} L|$ the channel can erase or introduce $w$ depending on whether $\delta$ or $l$ occurs in $\tau$. Hence, $L$ is not error-detecting for $\gamma$. Now if $|w| \geqslant|\operatorname{syn} L|$, a pumping lemma of the regular languages (see [19]) implies that there are words $x, y, z$ such that $w=x y z$, $1 \leqslant|y| \leqslant|\operatorname{syn} L|$, and $x y^{n} z \in L$ for all $n$ in $\mathbb{N}_{0}$. As $x y z$ and $x z$ are in $L$ the channel can transform one of these words to the other; therefore, $L$ is not error-detecting for $\gamma$.

\section{Error detection with finite delay}

Although error-detection is a basic property of a communications language, the process of decoding a message of such a language might require unbounded memory. This is because the decoder needs to see the entire message in order to decide whether it is correct. Moreover, the message is either accepted or rejected in its entirety. On the other hand, it is possible to define language and automaton (or transducer) properties that allow one to decode a message, possibly partially, using bounded memory $[12,6,2-4]$. This section introduces the code property of error-detection with finite delay which ensures that, as long as a sufficient number of consecutive codewords is received, the process of decoding those codewords can begin before receiving the rest of the message. On the other hand, the decoder can signal an error if it receives a part of the message which is not the concatenation of a sufficient number of codewords. In this case, the decoding process gets suspended and what follows depends on the communication protocol—usually involving retransmission techniques.

Definition 5. Let $\gamma$ be a channel.

(i) A code $K$ is said to have finite $\left(\gamma,{ }^{*}\right)$-detection delay, if there is a non-negative integer $d$ such that

$$
\begin{aligned}
& \forall v \in K, \forall z \in K^{d} X^{*}, \forall w \in K^{*}, \\
& v z \in\langle w\rangle_{\gamma} \rightarrow \exists u \in K^{*}, w=v u \text { and } z \in\langle u\rangle_{\gamma} .
\end{aligned}
$$


In this case, we say that $K$ has $(\gamma, *)$-detection delay $d$.

(ii) A coded language $L$ is error-detecting for $\gamma$ with finite delay, if it is error-detecting for $\gamma$ and the code $C_{L}$ has finite $(\gamma, *)$-detection delay. In this case, if $C_{L}$ has $(\gamma, *)$ detection delay $d$, for some $d \in \mathbb{N}_{0}$, we say that $L$ is error-detecting for $\gamma$ with delay $d$.

The property of finite $(\gamma, *)$-detection delay is analogous to the code property of finite decoding delay for error-free channels (see [2] where the term finite deciphering delay is used, or [3] where the term bounded deciphering delay is used). Specifically, a language $K$ is said to have finite decoding delay $d$ if

$$
\forall v, v^{\prime} \in K, \forall z \in K^{d} X^{*}, \quad v z \in v^{\prime} K^{*} \rightarrow v=v^{\prime} .
$$

Remark 1. Let $\gamma$ be a $P_{*}$-channel, let $d \in \mathbb{N}_{0}$, and let $K$ be a code that has $(\gamma, *)$ detection delay $d$. Then, the following statements hold true:

(i) The code $K$ has $(\gamma, *)$-detection delay $d+1$.

(ii) The code $K$ has finite decoding delay $d$.

Lemma 1. Let $K$ be a code, let $\gamma$ be a channel, and let $d \in \mathbb{N}_{0}$. If $K$ has $(\gamma, *)$ detection delay $d$ then the following property, $2_{\gamma}^{d}(n, K)$, holds for all $n \in \mathbb{N}_{0}$ :

$$
\begin{aligned}
& \forall v \in K^{n}, \forall z \in K^{d} X^{*}, \forall w \in K^{*}, \\
& v z \in\langle w\rangle_{\gamma} \rightarrow \exists u \in K^{*}, w=v u \text { and } z \in\langle u\rangle_{\gamma} .
\end{aligned}
$$

Proof. Assume that $K$ has $(\gamma, *)$-detection delay $d$. We use induction on $n \in \mathbb{N}_{0}$. First, it is easy to see that $\mathscr{Q}_{\gamma}^{d}(0, K)$ holds. Now assume $\mathscr{Q}_{\gamma}^{d}(n, K)$ holds for some $n \geqslant 0$. We show that $\mathscr{Q}_{\gamma}^{d}(n+1, K)$ holds too. Let $v \in K^{n+1}, z \in K^{d} X^{*}$, and $w \in K^{*}$ such that $v z \in\langle w\rangle_{\gamma}$. Then, $v=v_{1} v_{2}$ for some words $v_{1} \in K$ and $v_{2} \in K^{n}$. Moreover, as $v_{2} z \in K^{d} X^{*}$ and $K$ has $(\gamma, *)$-detection delay $d$, there is $u^{\prime} \in K^{*}$ such that $w=v_{1} u^{\prime}$ and $v_{2} z \in\left\langle u^{\prime}\right\rangle_{\gamma}$. Then, $2_{\gamma}^{d}(n, K)$ implies the existence of a message $u \in K^{*}$ such that $u^{\prime}=v_{2} u$ and $z \in\langle u\rangle_{\gamma}$. Thus, we have shown that $w=v_{1} u^{\prime}=v u$ and $z \in\langle u\rangle_{\gamma}$, for some $u \in K^{*}$, which implies that $\mathscr{Q}_{\gamma}^{d}(n+1, K)$ holds.

Example 6. The code $K_{1}$ of Example 5 has $(\gamma, *)$-detection delay 0 . This follows from the fact (shown in Example 5) that when a received message starts with a codeword $v$ in $K_{1}$ then this codeword is correctly transmitted. Then, as a consequence of $\mathscr{Q}_{\gamma}^{0}\left(3, K_{1}\right)$, if $v_{1} v_{2} v_{1} a a b a b b$ is a prefix of the received message, the words $v_{1}, v_{2}, v_{1}$ can be decoded correctly and an error is detected when $a a b a b b$ is encountered.

In [2], it is shown that if a language $K$ has finite decoding delay then $K$ is a code. Motivated by this statement, we consider next the question of whether a code with finite $(\gamma, *)$-detection delay is $(\gamma, *)$-detecting. 
Theorem 2. Let $\gamma$ be a $P_{*}$-channel, let $d \in \mathbb{N}_{0}$, and let $K$ be a code with $(\gamma, *)$-detection delay $d$. Then, the following statements hold true:

(i) The code $K$ is $(\gamma, *)$-detecting if and only if it is $(\gamma, d)$-detecting.

(ii) If $|K|>1$ then $K$ is $(\gamma, *)$-detecting.

Proof. (i) The 'only if' part follows immediately from Proposition 1(ii). For the 'if' part, assume $K$ is $(\gamma, d)$-detecting and consider messages $w_{1}, w_{2} \in K^{*}$ with $w_{1} \in\left\langle w_{2}\right\rangle_{\gamma}$. There is $m \in \mathbb{N}_{0}$ such that $w_{1}=v_{1} \cdots v_{m}$ and each $v_{i}$ is in $K$. If $m \leqslant d$ then $w_{2}=w_{1}$ as required. Now assume that $m=n+d$ for some $n>0$. As $w_{1} \in K^{n} K^{d} X^{*}$ and $w_{1} \in\left\langle w_{2}\right\rangle_{\gamma}$, property $\mathscr{Q}_{\gamma}^{d}(n, K)$ of Lemma 1 implies $w_{2}=v_{1} \cdots v_{n} u$ and $v_{n+1} \cdots v_{n+d} \in\langle u\rangle_{\gamma}$ for some message $u \in K^{*}$. On the other hand, $v_{n+1} \cdots v_{n+d} \in K^{\leqslant d}$ implies $u=v_{n+1} \cdots v_{n+d}$. Hence, $w_{2}=w_{1}$ as required.

(ii) Assume $|K|>1$. By the first part, it is sufficient to show that $K$ is $(\gamma, d)$-detecting. Let $v \in K^{\leqslant d}$ and $w \in K^{*}$ such that $v \in\langle w\rangle_{\gamma}$. Then, $v=[\varphi]$ and $w=[\psi]$ for some factorizations $\varphi, \psi$ over $K$ with $|\varphi| \leqslant d$. Choose a codeword $x$ such that

$$
x \in \begin{cases}K \backslash\{\psi(|\varphi|)\} & \text { if }|\psi|>|\varphi| ; \\ K \backslash\{\varphi(|\psi|)\} & \text { if }|\varphi|>|\psi| ; \\ K & \text { if }|\varphi|=|\psi| .\end{cases}
$$

As $v \in\langle w\rangle_{\gamma}, \mathscr{P}_{2}$ implies $v x^{d} \in\left\langle w x^{d}\right\rangle_{\gamma}$. As $K$ has $(\gamma, *)$-detection delay $d$, there is $u \in K^{*}$ such that $w x^{d}=v u$ and $x^{d} \in\langle u\rangle_{\gamma}$. Hence, $[\varphi] u=[\psi] x^{d}$. If $|\varphi|=|\psi|$ then, as $K$ is a code, $\varphi=\psi$ and, therefore, $w=v$ as required. If $|\varphi|>|\psi|$ then $d>0$ and $\varphi(|\psi|)=x$ which contradicts $x \in K \backslash\{\varphi(|\psi|)\}$. Finally, if $|\psi|>|\varphi|$ then $u \in \psi(|\varphi|) K^{*}$. As $x^{d} \in\langle u\rangle_{\gamma}$, one has $x^{d+1} \in\langle u x\rangle_{\gamma}$. But, as $K$ has $(\gamma, *)$-detection delay $d, u x \in x K^{*}$ which contradicts $x \in K \backslash\{\psi(|\varphi|)\}$. Hence, $K$ is $(\gamma, d)$-detecting.

We note that the second statement of Theorem 2 is not true in general if $|K|=1$. For example, consider the alphabet $X=\{a, b\}$ and the channel $\gamma=\{(a \mid a),(\lambda \mid a),(b \mid b)\}^{*}$, where the concatenation between two pairs of words is defined naturally: $\left(y_{1}^{\prime} \mid y_{1}\right)$ $\left(y_{2}^{\prime} \mid y_{2}\right)=\left(y_{1}^{\prime} y_{2}^{\prime} \mid y_{1} y_{2}\right)$. Then, $\gamma$ is a $P_{*}$-channel and, as $\lambda \in\langle a\rangle_{\gamma}$, the code $\{a\}$ is not $(\gamma, *)$-detecting. On the other hand, $\{a\}$ has $(\gamma, *)$-detection delay 0 .

The following lemma gives an expression for the maximum number of deletion errors that can occur when a word $w$ is communicated through an SID-channel $\tau(m, \ell)$ that permits deletions.

Lemma 2. Let $m, \ell \in \mathbb{N}$ with $\ell>m$ and let $D_{m, \ell}: \mathbb{N}_{0} \rightarrow \mathbb{N}_{0}$ be such that, for all $n \in \mathbb{N}_{0}, D_{m, \ell}(n)=\lfloor n / \ell\rfloor m+\min \left(m, r_{\ell}(n)\right)$ where $r_{\ell}(n)$ is the remainder of the integer division $n / \ell$. Let $\tau$ be an error type that contains the deletion type and let $\gamma=\tau(m, \ell)$. For every word $w$ the following statements hold true:

(i) If $z \in\langle w\rangle_{\gamma}$ then $|w|-|z| \leqslant D_{m, \ell}(|w|)$.

(ii) For every non-negative integer $k, k \leqslant D_{m, \ell}(|w|)$ if and only if $\langle w\rangle_{\gamma} \cap X^{|w|-k} \neq \emptyset$. 
Proof. Let $n=|w|$ and let $q=\lfloor n / \ell\rfloor$. Then $w=w_{1} \cdots w_{q} s$ for some words $w_{i} \in X^{\ell}$ and $s \in X^{r(n)}$.

(i) If $z \in\left\langle w_{1} \cdots w_{q} s\right\rangle_{\gamma}$, property $\mathscr{P}_{1}$ implies that $z=w_{1}^{\prime} \cdots w_{q}^{\prime} s^{\prime}$ for some words $w_{i}^{\prime}, s^{\prime}$ with $w_{i}^{\prime} \in\left\langle w_{i}\right\rangle_{\gamma}$ and $s^{\prime} \in\langle s\rangle_{\gamma}$. As the channel $\gamma$ permits at most $m$ deletions in a word of length $\ell$ or less, it follows that $\left|w_{i}\right|-\left|w_{i}^{\prime}\right| \leqslant m$ and $|s|-\left|s^{\prime}\right| \leqslant \min \left(m, r_{\ell}(n)\right)$. Hence, $|w|-|z| \leqslant m q+\min \left(m, r_{\ell}(n)\right)$.

(ii) The 'if' part follows easily from the first statement of the lemma. For the 'only if' part, let $k$ be a non-negative integer not exceeding $D_{m, \ell}(n)$ and let $p=\lfloor k / m\rfloor$. Then, $m p+r_{m}(k) \leqslant q m+\min \left(m, r_{\ell}(n)\right)$. If $p \leqslant q$ then, for $y=w_{p+1} \cdots w_{q} s$, one has $r_{m}(k) \leqslant|y|$ and a word $z$ can be obtained by deleting in $w$ the first $m$ symbols of every $w_{j}$, for $j=1, \ldots, p$, and the first $r_{m}(k)$ symbols of $y$. Hence, as $k=m p+r_{m}(k)$, one has $z \in\langle w\rangle_{\gamma} \cap X^{n-k}$. If $p>q$ then $p=q+1, r_{m}(k)=0$, and $r_{\ell}(n) \geqslant m$. In this case, $s \in X^{m} X^{*}$ and a word $z$ can be obtained by deleting in $w$ the first $m$ symbols of every $w_{i}$, for $i=1, \ldots, q$, and the first $m$ symbols of $s$. Hence, as $k=(q+1) m$, one has $z \in\langle w\rangle_{\gamma} \cap X^{n-k}$.

Next it is shown that if an SID-channel permits insertions then there is no coded language which is error-detecting for that channel with delay 0 .

Theorem 3. Let $m, \ell \in \mathbb{N}$, let $\tau$ be an error type, and let $L$ be a coded language which is error-detecting for $\tau(m, \ell)$ with delay 0 . Then, the following statements hold true:

(i) The insertion type is not contained in $\tau$.

(ii) If $\ell \geqslant 2 m$ and $\tau$ contains the deletion type then $v X^{*} v \cap L=\emptyset$ for all $v \in X^{+}$with $|v| \leqslant m$.

Proof. (i) Assume $L$ is error-detecting for $\gamma$ with delay 0 , where $\gamma=\tau(m, \ell)$, but suppose that $\tau$ contains the insertion type. Theorem 1(i) implies that the alphabet, $X$, of $L$ contains at least two symbols and that no symbol of $X$ is in $L$. Let $w$ be any word in $C_{L}$. Then $w=a x b$ for some $a, b \in X$ and $x \in X^{*}$. Let $c$ be any symbol in $X \backslash\{b\}$ and let $n=|a x b|$. As the length of the word $(a x b)^{1+\ell}$ is $n(1+\ell),(a x b)^{1+\ell}=y_{1} b_{1} \cdots y_{n} b_{n}$ where $b_{i} \in X, y_{i} \in X^{\ell}$ and $b_{n}=b$. As $\tau(m, \ell)$ permits insertions, one has

$$
\left(a x b^{m} b\right)\left(y_{1} c^{m} b_{1}\right) \cdots\left(y_{n} c^{m} b_{n}\right) \in\left\langle a x b(a x b)^{1+\ell}\right\rangle_{\gamma} .
$$

As $L$ is error-detecting for $\gamma$ with delay 0 , one has $z \in\left\langle(a x b)^{1+\ell}\right\rangle_{\gamma}$, where $z$ is the word $b^{m} y_{1} c^{m} b_{1} \cdots y_{n} c^{m} b_{n}$. In obtaining $z$ from $y_{1} b_{1} \cdots y_{n} b_{n}$ via $\gamma$, consider for every $i=1, \ldots, n$ the number $k_{i}$ of inserted symbols in $y_{i} b_{i}$ to the left of $b_{i}$, and the number $k_{n+1}$ of inserted symbols at the end of $y_{1} b_{1} \cdots y_{n} b_{n}$. Then, $0 \leqslant k_{j} \leqslant m$ for all $j=1, \ldots, n+1$. On the other hand, as $|z|=\left|(a x b)^{1+\ell}\right|+(n+1) m$, one has $k_{1}+\cdots+k_{n+1} \geqslant(n+1) m$ (with equality when no deletion errors occur); therefore, $k_{n+1}=m$. This implies that the last $m$ symbols of $z$, namely $c^{m-1} b_{n}$, are inserted and the preceding symbol, namely $c$, is the last symbol of $(a x b)^{1+\ell}$. Hence, $c=b$ which is a contradiction. 
(ii) Assume that the language $L$ is a error-detecting for $\gamma$ with delay 0 , where $\gamma=\tau(m, \ell)$, but suppose there is a word $w$ in $L$ such that $w=v x v$ for some words $x$ and $v$ with $1 \leqslant|v| \leqslant m$. Then, $w \in C_{L}^{+}$. Let $n=|w|$ and let $k=|v|$. Then $w^{\ell}=y_{1} v_{1} \cdots y_{n} v_{n}$ for some words $y_{1}, \ldots, y_{n} \in X^{\ell-m}$ and $v_{1}, \ldots, v_{n} \in X^{m}$. As $\left|y_{1}\right| \geqslant k$ and $y_{1}$ is a prefix of $w^{\ell}$, there are words $u \in X^{m-k}$ and $x_{1} \in X^{\ell-2 m}$ such that $y_{1}=v u x_{1}$. Moreover, as $\left|v_{1}\right|=m$, there are words $u_{1} \in X^{m-k}$ and $u_{2} \in X^{k}$ such that $v_{1}=u_{1} u_{2}$. Hence,

$$
w^{1+\ell}=(v x v)\left(v u x_{1} u_{1} u_{2}\right) y_{2} v_{2} \cdots y_{n} v_{n} .
$$

Now consider the word $z=v x v x_{1} u_{1} y_{2} \cdots y_{n}$ which can be obtained by deleting in $w^{1+\ell}$ the suffix $v$ of the first $w$ and the words $u, u_{2}, v_{2}, \ldots, v_{n}$. Then, $z \in\left\langle w^{1+\ell}\right\rangle_{\gamma}$ and Lemma 1 implies $y \in\left\langle w^{\ell}\right\rangle_{\gamma}$, where $y=x_{1} u_{1} y_{2} \cdots y_{n}$. By Lemma 2, one has

$$
\begin{aligned}
& \left|w^{\ell}\right|-|y| \leqslant D_{m, \ell}\left(\left|w^{\ell}\right|\right) \\
& \quad \Rightarrow n \ell-(\ell-2 m+m-k+(n-1)(\ell-m)) \leqslant m n \Rightarrow k \leqslant 0
\end{aligned}
$$

which is a contradiction. Hence, $v X^{*} v \cap L=\emptyset$.

\section{Error-detecting languages and codes for SID channels}

In this section we consider certain error-detecting capabilities of some known classes of codes. There are cases where, due to the characteristics of the codes used, $(\gamma, 1)$ detection is sufficient to ensure $(\gamma, *)$-detection. On the other hand, for some classes of codes, $(\gamma, 1)$-detection is provided for free. Moreover, in this section, we provide a construction of a maximal error-detecting language for the channel $\delta(m, \ell)$ and give an example of a uniform coded language which is error-detecting for $\delta(m, \ell)$ and $l(m, \ell)$ with finite delay. The first result concerns the channel $\sigma(m, \ell)$ that involves only substitution errors. This result justifies the use of uniform codes for such channels.

Proposition 2. Let $m, \ell \in \mathbb{N}$, let $K$ be a uniform code, and let $\gamma$ be the channel $\sigma(m, \ell)$. Then, $K$ is $(\gamma, *)$-detecting if and only if it is $(\gamma, 1)$-detecting.

Proof. The 'only if' part follows immediately from Proposition 1(ii). Now assume that $K$ is a uniform code of length $n \in \mathbb{N}$ and that $K$ is $(\gamma, 1)$-detecting. Let $w_{1}, w_{2}$ be messages in $K^{*}$ such that $w_{1} \in\left\langle w_{2}\right\rangle_{\gamma}$. Then, there are factorizations $\kappa_{1}, \kappa_{2}$ over $K$ such that $\left[\kappa_{1}\right]=w_{1}$ and $\left[\kappa_{2}\right]=w_{2}$. Property $\mathscr{P}_{1}$ implies that there is a factorization $\psi$ which is $\gamma$-admissible for $\kappa_{2}$ such that $w_{1}=[\psi]$ and $\psi(i) \in\left\langle\kappa_{2}(i)\right\rangle_{\gamma}$ for all $i \in I_{\psi}=I_{\kappa_{2}}$. As $\gamma$ permits only substitutions, one has $|\psi(i)|=n$ for all $i \in I_{\kappa_{2}}$. Hence, $|[\psi]|=n\left|\kappa_{2}\right|$. On the other hand, $\left|w_{1}\right|=n\left|\kappa_{1}\right|$; therefore, $\left|\kappa_{1}\right|=\left|\kappa_{2}\right|=|\psi|$ which implies $\psi=\kappa_{1}$. Now as $\kappa_{1}(i) \in\left\langle\kappa_{2}(i)\right\rangle_{\gamma}$ and $K$ is $(\gamma, 1)$-detecting, it follows that $\kappa_{1}(i)=\kappa_{2}(i)$ for all $i \in I_{\kappa_{1}}$. Hence, $w_{1}=w_{2}$.

A similar statement follows about finite solid codes for the channel $\sigma \odot \imath \odot \delta(1, \ell)$. A language $K$ is a solid code, if it is an infix and overlap-free language; that is, 
$K \cap\left(X^{*} K X^{+} \cup X^{+} K X^{*}\right)=\emptyset$ and, for all $u, v \in X^{+}$and $x \in X^{*}, \quad v x, x u \in K$ implies $x=\lambda$. Some interesting decoding capabilities of solid codes are discussed in [9]. Recent results on solid codes can be found in [7] and [11].

The proof of the following result is based on a special property of the assumed type of solid codes. Let $K$ be a code and let $\gamma$ be a $P_{*}$-channel. A factorization $\psi$ is said to be $(\gamma, K)$-corrupted, if it is $\gamma$-admissible for some factorization $\kappa$ over $K$ and $\kappa \neq \psi$. Thus, $[\psi] \in\langle[\kappa]\rangle_{\gamma}$ and there is at least one factor $\psi(i)$ of $\psi$ which is not equal to its corresponding factor $\kappa(i) \in K$. The property we need is as follows:

$\mathscr{P}(\gamma, K)$ : If $\psi$ is a $(\gamma, K)$-corrupted factorization then $[\psi] \notin K^{*}$.

One can verify that every code satisfying $\mathscr{P}(\gamma, K)$ must be a $(\gamma, *)$-detecting code.

Theorem 4. Let $\ell \in \mathbb{N}$, let $\gamma$ be the channel $\sigma \odot \imath \odot \delta(1, \ell)$, and let $K$ be a finite solid code with maxlen $K \leqslant \ell$. Then, $K$ is $(\gamma, *)$-detecting if and only if it is $(\gamma, 1)$-detecting.

Proof. The 'only if' part follows immediately from Proposition 1(ii). Now assume that $K$ is $(\gamma, 1)$-detecting. We show that $\mathscr{P}(\gamma, K)$ holds. Let $\kappa$ be a factorization over $K$ and let $\psi$ be $\gamma$-admissible for $\kappa$ such that $\psi \neq \kappa$. Then, $|\kappa|=|\psi|>0$. Now suppose that $[\psi] \in K^{*}$; that is, $[\psi]=[\mu]$ for some factorization $\mu$ over $K$. If $|\mu|=0$ then $[\mu]=\lambda \in\langle[\kappa]\rangle_{\gamma}$ which contradicts the fact that $K$ is $(\gamma, 1)$-detecting. Hence, $|\mu|>0$.

Let $k=|\kappa|=|\psi|$ and $m=|\mu|$. Then, $[\psi]=\psi(0) \cdots \psi(k-1)=\mu(0) \cdots \mu(m-1)$. As $\kappa \neq \psi$, there is a minimum $p \in I_{\kappa}$ such that $\kappa(p) \neq \psi(p)$. Then, $[\psi]=\kappa(0) \cdots \kappa(p-$ $1) \psi(p) \cdots \psi(k-1)$ and, as $K$ is a prefix code, $\kappa(i)=\mu(i)$ for all $i<p$. Hence, $\psi(p) \cdots \psi(k-1)=\mu(p) \cdots \mu(m-1)$. Now, for all $j$ in $\{p, p+1, \ldots, k-1\}$ one has

$$
\psi(j)= \begin{cases}x_{j} y_{j} & \text { if } \kappa(j)=x_{j} a_{j} y_{j} \text { with } a_{j} \in X \text { deleted; } \\ x_{j} a_{j} y_{j} & \text { if } \kappa(j)=x_{j} y_{j} \text { with } a_{j} \in X \text { inserted, or } \\ & \kappa(j)=x_{j} b_{j} y_{j} \text { with } b_{j} \in X \text { substituted with } a_{j} \in X \\ \kappa(j) & \text { if no error occurs. }\end{cases}
$$

Of course, when $j=p, \psi(j) \neq \kappa(j)$. For the lengths of $\mu(p)$ and $\psi(p)$ we distinguish three cases which all lead to contradictions due to the fact that $K$ is a $(\gamma, 1)$-detecting solid code.

First, assume $|\mu(p)|>|\psi(p)|$. Then, $\mu(p)=\psi(p) \cdots \psi(r) w$ where $p \leqslant r$ and $w$ is either equal to $\psi(r+1)$ or to a non-empty proper prefix of $\psi(r+1)$. The former case implies $\mu(p) \in\left\langle K^{2} K^{*}\right\rangle_{\gamma} \cap K$ which is impossible. Hence, $0<|w|<|\psi(r+1)|$ and $\psi(r+1)=w s$ with $s \in X^{+}$. The case $\psi(r+1)=\kappa(r+1)$ is not possible, as otherwise $w$ would be a proper suffix of $\mu(p)$ and a proper prefix of $\kappa(r+1)$. Thus, $\psi(r+1)$ is of the form $x_{r+1} y_{r+1}$ or $x_{r+1} a_{r+1} y_{r+1}$. If $|w| \leqslant\left|x_{r+1}\right|$ the overlap-freeness of $K$ is violated again. Hence, $w s=x_{r+1} y_{r+1}$ or $w s=x_{r+1} a_{r+1} y_{r+1}$, and $|w|>\left|x_{r+1}\right|$. It follows then that $\mu(p+1)$ either is contained in $y_{r+1}$ or it starts with a proper suffix of $y_{r+1}$ which is a contradiction. 
Now assume $|\mu(p)|<|\psi(p)|$. Then, $\psi(p)=\mu(p) s$ where $s \in X^{+}$and $m>p$. As $K$ is an infix code, it must be $|\mu(p)|>\left|x_{p}\right|$ and, therefore, $|s| \leqslant\left|y_{p}\right|$. Then, however, $\mu(p+1)$ is either contained in $y_{p}$ or it starts with a suffix of $y_{p}$. Finally, the case $|\mu(p)|=|\psi(p)|$ is also impossible, as it violates the fact that $K$ is $(\gamma, 1)$-detecting.

Example 7. The code $K_{1}$ of Example 5 is a $(\gamma, 1)$-detecting solid code, where $\gamma=\sigma \odot$ $\imath \odot \delta(1,7)$. Hence, Theorem 4 implies that $K_{1}$ is $(\gamma, *)$-detecting as well.

Let us consider now certain classes of shuffle codes, as they provide error-detecting capabilities for SID-channels that involve either insertions or deletions. A language $K$ is a prefix-shuffle code of index $n \in \mathbb{N}$, if $x_{0} \cdots x_{n-1} \in K$ and $x_{0} y_{0} \cdots x_{n-1} y_{n-1} \in K$ imply $y_{0}=\cdots=y_{n-1}=\lambda$, for all words $x_{i}$ and $y_{i}$ in $X^{*}$. Let $\mathrm{PS}_{n}$ be the class of prefix-shuffle codes of index $n$. Then, $\mathrm{PS}_{n+1} \subseteq \mathrm{PS}_{n}$ for all $n \in \mathbb{N}$. The class $\mathrm{OS}_{n}$ of outfix-shuffle codes of index $n$ is defined analogously: $x_{0} \cdots x_{n} \in K$ and $x_{0} y_{0} \cdots x_{n-1} y_{n-1} x_{n} \in K$ imply $y_{0}=\cdots=y_{n-1}=\lambda$. Again, one has $\operatorname{OS}_{n+1} \subseteq \mathrm{OS}_{n}$ for all $n \in \mathbb{N}$. We refer the reader to [9] for further results on shuffle codes.

Proposition 3. Let $m, \ell \in \mathbb{N}$ with $m<\ell$, and let $K$ be a code with minlen $K>m$ and maxlen $K \leqslant \ell$.

(i) If $K$ is outfix-shuffle of index $m$ then it is error-detecting for $\imath(m, \ell)$ and for $\delta(m, \ell)$.

(ii) If $K$ is prefix-shuffle of index $m+1$ then it is $(\gamma, 1)$-detecting, where $\gamma=$ $\imath(m, \ell)$.

Proof. (i) Let $\gamma=\delta(m, \ell)$. As minlen $K>m, \lambda \notin\langle K\rangle_{\gamma}$. As $\gamma$ permits only deletions, $\langle\lambda\rangle_{\gamma} \cap K=\emptyset$. Moreover, if $x \in K$ and $z \in\langle x\rangle_{\gamma}$ there is $k \in \mathbb{N}_{0}$ with $k \leqslant m$ such that $x=x_{0} a_{0} \cdots x_{k-1} a_{k-1} x_{k}$ and $z=x_{0} \cdots x_{k-1} x_{k}$, where $a_{0}, \ldots, a_{k-1} \in X$ are the deleted symbols and $x_{0}, \ldots, x_{k} \in X^{*}$. From this observation and the fact $\operatorname{OS}_{m} \subseteq \operatorname{OS}_{k}$ for $k \leqslant m$, it follows that if $K$ is outfix-shuffle of index $m$ then it is error-detecting for $\delta(m, \ell)$. Using a similar argument, one can show that $K$ is also error-detecting for $\imath(m, \ell)$.

(ii) Let $K$ be prefix-shuffle of index $m+1$ and let $w_{1} \in K \cup\{\lambda\}$ and $w_{2} \in K^{*}$ such that $w_{1} \in\left\langle w_{2}\right\rangle_{\gamma}$. As minlen $K>m$ and $\gamma$ permits at most $m$ insertions in any $\ell$ or less consecutive symbols of $w_{2}$, it follows that when one of $w_{1}$ and $w_{2}$ is empty they must both be empty. Now assume $w_{1} \in K$ and $w_{2} \in K^{n}$ for some $n$ in $\mathbb{N}$. Then, $w_{2}=[\kappa]$ and $w_{1}=[\psi]$, where $\kappa$ is a factorization over $K$ of length $n$ and $\psi$ is $\gamma$-admissible for $\kappa$. We show that $w_{1}=\kappa(0)$. As $\psi(0) \in\langle\kappa(0)\rangle_{\gamma}$ and $|\kappa(0)| \leqslant \ell$, at most $m$ insertions can occur in $\kappa(0)$. More specifically, let $k$ be the number of insertions in $\kappa(0)$ and let $a_{0}, \ldots, a_{k-1} \in X$ be the symbols inserted. Then, $0 \leqslant k \leqslant m$ and, $\psi(0)=x_{0} a_{0} \cdots x_{k-1} a_{k-1} x_{k}$ and $\kappa(0)=x_{0} \cdots x_{k-1} x_{k}$ for some words $x_{0}, \ldots, x_{k-1}, x_{k}$. Now $[\psi]=\psi(0) s$ and $s \in\langle\kappa(1) \cdots \kappa(n-1)\rangle_{\gamma}$, for some $s$ in $X^{*}$. Hence, $w_{1}=x_{0} a_{0} \cdots$ $x_{k-1} a_{k-1} x_{k} s$. As $K$ is prefix-shuffle of index $m+1$, it is also prefix-shuffle of index $k+1$ and, therefore, $w_{1}=\kappa(0)$ which implies $k=0$ and $s=\lambda$. Moreover, $\kappa(1) \cdots \kappa(n-1)=\lambda$ implies $n=1$ and $w_{2}=\kappa(0)$. Hence, $w_{1}=w_{2}$ as required. 
We note that a code satisfying the premises of Proposition 3 is not necessarily $(\gamma, *)$-detecting. For example, the code $K_{0}$ of Example 4 is prefix-shuffle of index 2 and $(\gamma, 1)$-detecting, where $\gamma=\imath(1,3)$. But $K_{0}$ is not $(\gamma, *)$-detecting.

In the rest of the section we give an example of a uniform coded language $M$ which is error-detecting for $\delta(m, \ell)$ and for $\imath(m, \ell)$ with finite delay, and construct an infinite non-coded language $L$ which is maximal error-detecting for $\delta(m, \ell)$ and whose redundancy is asymptotically optimal. For the language $M$ we follow the approach of separator words used in [13] for constructing uniform codes which are error-correcting with finite delay in the presence of certain SID-channel errors. Moreover, we consider the problem of estimating the redundancy imposed by the error-detecting capabilities of $M$ and $L$. Normally, the redundancy of a finitely coded language is expressed in terms of the size and the average word length of the code that generates the language. As the language $L$ is not coded, however, we consider the redundancies of the infinite languages $M$ and $L$ as follows (see [14,9]): Let $W=\left\{w_{n} \mid n \in \mathbb{N}_{0}\right\}$ be an infinite language such that $\left|w_{n}\right| \leqslant\left|w_{n+1}\right|$ for all $n \in \mathbb{N}_{0}$. Then, the redundancy of $W$ is the function defined by $\varrho_{W}(n)=\left|w_{n}\right|-\left\lfloor\log _{r} n\right\rfloor-1$ for all $n \in \mathbb{N}_{0}$, where $r$ is the size of the alphabet $X$. Intuitively, assuming an unbounded number of possible objects (or messages), $\varrho_{W}(n)$ gives the number of extra alphabet symbols required to represent the object $n$ using the word $w_{n}$ as opposed to using the $r$-ary representation of the number $n$. Usually, as $n \rightarrow \infty$, one gives asymptotic estimates for $\varrho_{W}(n)$. For two functions $f(n)$ and $g(n)$ we say that

- $f(n)$ is asymptotically upper-bounded by $g(n)$, if $\lim \sup (f(n) / g(n)) \leqslant 1$.

- $f(n)$ is asymptotically lower-bounded by $g(n)$, if $\lim \inf (f(n) / g(n)) \geqslant 1$.

- $f(n)$ is asymptotically equal to $g(n)$, if $f(n)$ is asymptotically upper- and lowerbounded by $g(n)$; or equivalently, if $\lim (f(n) / g(n))=1$.

- $f(n)$ is strictly asymptotically upper-bounded by $g(n)$, if $\lim \sup (f(n) / g(n))<1$. In this case, $f(n)$ is asymptotically upper-bounded by $g(n)$, but it is not asymptotically equal to $g(n)$.

We also note that, in estimating the redundancy of an infinite language $W$, the following fact is useful

$$
\forall n \in \mathbb{N}_{0}, \quad N_{W}\left(\left|w_{n}\right|-1\right) \leqslant n<N_{W}\left(\left|w_{n}\right|\right),
$$

where $N_{W}: \mathbb{N}_{0} \rightarrow \mathbb{N}_{0}$ is the cumulative density function of $W$ such that $N_{W}(k)$ is the number of words in $W$ whose length is at most $k$.

Lemma 3. For every languages $L$ and $L^{\prime}$, if the redundancy of $L^{\prime}$ is strictly asymptotically upper-bounded by the redundancy of $L$ then the set $\left\{t \in \mathbb{N}_{0} \mid N_{L^{\prime}}(t) \leqslant N_{L}(t)\right\}$ is finite.

Proof. Assume that the languages $L$ and $L^{\prime}$ satisfy the premise, but suppose there is a subsequence $\left\{t_{k}\right\}_{k \in \mathbb{N}_{0}}$ of $\mathbb{N}_{0}$ such that $N_{L^{\prime}}\left(t_{k}\right) \leqslant N_{L}\left(t_{k}\right)$ for all $k$. Let $L=\left\{w_{n} \mid n \in \mathbb{N}_{0}\right\}$ and $L^{\prime}=\left\{w_{n}^{\prime} \mid n \in \mathbb{N}_{0}\right\}$ such that $\left|w_{n}\right| \leqslant\left|w_{n+1}\right|$ and $\left|w_{n}^{\prime}\right| \leqslant\left|w_{n+1}^{\prime}\right|$ for all $n$. Let $f(n)=$ $\varrho_{L^{\prime}}(n) / \varrho_{L}(n)$, for all $n$, and let $s=\lim \sup f(n)$ with $s<1$. First we show that the set 
$\left\{n \in \mathbb{N}_{0} \mid f(n) \geqslant 1\right\}$ is infinite. For each $k$, let $n_{k}=\max \left\{n|| w_{n} \mid \leqslant t_{k}\right\}$. Then, $\left|w_{n_{k}+1}\right|>t_{k}$ and $N_{L}\left(t_{k}\right)=1+n_{k}$. Moreover, $N_{L^{\prime}}\left(t_{k}\right)<n_{k}$ which implies $N_{L^{\prime}}\left(t_{k}\right)<N_{L^{\prime}}\left(\left|w_{n_{k}}^{\prime}\right|\right)$ using (1). As $N_{L^{\prime}}$ is monotonically increasing, one has $t_{k} \leqslant\left|w_{n_{k}}^{\prime}\right|$. Hence, $\left|w_{n_{k}}\right| \leqslant\left|w_{n_{k}}^{\prime}\right|$ for all $k$ which implies that $\left\{n \in \mathbb{N}_{0} \mid f(n) \geqslant 1\right\}$ is infinite.

By the assumption about $s$, the set $\left\{n \in \mathbb{N}_{0} \mid f(n)>s+\varepsilon\right\}$ is finite, for all $\varepsilon>0$. On the other hand, $\left\{n \in \mathbb{N}_{0} \mid f(n)>1-\varepsilon\right\}$ is infinite, for all $\varepsilon>0$, and a contradiction arises when one considers $\varepsilon=(1-s) / 2$.

Lemma 4. Let $p, s \in X^{*}$ and let $k \in \mathbb{N}$. The redundancy of the language $\left(p X^{k} s\right)^{*}$ is asymptotically equal to $|p s| / k \log _{r} n$, where $n \rightarrow \infty$ and $r=|X|$.

Proof. Let $L=\left(p X^{k} s\right)^{*}$, let $m=|p s|$, and let $\ell$ be a non-negative integer. As the lengths of the words in $L$ are multiples of $m+k$, one has $N_{L}(\ell)=\sum_{i=0}^{q} r^{\mathrm{i} k}$, where $q=\lfloor\ell /(m+k)\rfloor$. Hence, $N_{L}(\ell)=\left(r^{k(q+1)}-1\right) /\left(r^{k}-1\right)$. Now assume $L=\left\{w_{n} \mid n \in \mathbb{N}_{0}\right\}$ with $\left|w_{n}\right| \leqslant\left|w_{n+1}\right|$ for all $n \in \mathbb{N}_{0}$. Then, $N_{L}\left(\left|w_{n}\right|-1\right) \leqslant n$ and $n<N_{L}\left(\left|w_{n}\right|\right)$. The first inequality implies

$$
\frac{r^{k(q+1)}-1}{r^{k}-1} \leqslant n \Rightarrow q \leqslant \frac{1}{k} \log _{r}\left(n\left(r^{k}-1\right)+1\right)-1,
$$

where $q=\left\lfloor\left(\left|w_{n}\right|-1\right) /(m+k)\right\rfloor$. It follows then that $\left|w_{n}\right|<(m+k) / k \log _{r} n+\beta_{2}$ for some real constant $\beta_{2}$. Similarly, one can verify that the second inequality implies $\left|w_{n}\right|>(m+k) / k \log _{r} n+\beta_{1}$ for some real constant $\beta_{1}$. Hence, the redundancy of $L$ is asymptotically equal to $\left|w_{n}\right|-\log _{r} n=(m / k) \log _{r} n$.

Proposition 4. Let $m, \ell \in \mathbb{N}$ with $\ell>2 m+1$, let $a, b \in X$ with $a \neq b$, and let $M=$ $\left(a^{m} X^{\ell-2 m-1} b\right)^{*}$. Then, the following statements hold true:

(i) The language $M$ is error-detecting for $\delta(m, \ell)$ with delay 0.

(ii) The language $M$ is error-detecting for $1(m, \ell)$ with delay 1.

(iii) The redundancy of $M$ is asymptotically equal to $(m+1) /(\ell-2 m-1) \log _{r} n$, where $n \rightarrow \infty$ and $r$ is the size of the alphabet $X$.

Proof. (i) Let $\gamma=\delta(m, \ell)$, let $w \in M$ and assume $a^{m} x b z \in\langle w\rangle_{\gamma}$ for some $x \in X^{\ell-2 m-1}$ and $z \in X^{*}$. Then $w=[\varphi]$ for some factorization $\varphi$ over $C_{M}=a^{m} X^{\ell-2 m-1} b$. By $\mathscr{P}_{1}$, there is a factorization $\varphi^{\prime}$ of $a^{m} x b z$ which is $\gamma$-admissible for $\varphi$. First we show that $\varphi(0) \neq \varphi^{\prime}(0)$ is impossible. Indeed, this implies that $\varphi^{\prime}(0)$ can be obtained from $\varphi(0)$ using exactly $d$ deletions, where $d=|\varphi(0)|-\left|\varphi^{\prime}(0)\right|>0$. Moreover, in this case, $|\varphi| \geqslant 2$. As $\left|\varphi(0) a^{m}\right|=\ell$, the number of deletions in the prefix $a^{m}$ of $\varphi(1)$ is at most $m-d$ which implies that $\varphi^{\prime}(0) a^{d}$ is a prefix of $a^{m} x 1 z$. There is a contradiction, however, when we note that $\left|\varphi^{\prime}(0) a^{d}\right|=\left|a^{m} x b\right|$ and $a \neq b$. Hence, $\varphi(0)=\varphi^{\prime}(0)$ which implies $[\varphi]=a^{m} x b u$ and $z \in\langle u\rangle_{\gamma}$, where $u=\varphi(1) \cdots \varphi(|\varphi|-1)$.

(ii) Let $\gamma=\imath(m, \ell)$, let $w \in M$ and assume $a^{m} x_{1} b a^{m} x_{2} b y \in\langle w\rangle_{\gamma}$ for some $x_{1}, x_{2} \in$ $X^{\ell-2 m-1}$ and $y \in X^{*}$. Then $w=[\varphi]$ for some factorization $\varphi$ over $C_{M}=a^{m} X^{\ell-2 m-1} b$. Let $z=a^{m} x_{1} b a^{m} x_{2} b y$ and let $n$ be the number of insertions to the left of the suffix $b$ of $\varphi(0)$. Then, $z \in X^{\ell-m-1+n} b X^{*}$. If $n \geqslant 1$ then, as $n \leqslant m, z \in a^{m} x_{1} b a^{n} X^{*}$ which im- 
plies $z \in X^{\ell-m+n-1} a X^{*} \cap X^{\ell-m-1+n} b X^{*}$; a contradiction. Hence, $n=0$ and, therefore, $[\varphi]=a^{m} x_{1} b u$ and $a^{m} x_{2} b y \in\langle u\rangle_{\gamma}$, where $u=\varphi(1) \cdots \varphi(|\varphi|-1)$.

(iii) The statement follows from Lemma 4.

Theorem 5. Let $m, \ell \in \mathbb{N}$ with $\ell>m$ and let $L=\bigcup_{k=0}^{\infty} X^{h_{k}}$, where $h_{0}=0$ and $h_{k+1}=h_{k}$ $+1+m+m\left\lfloor h_{k} /(\ell-m)\right\rfloor$. Then, the following statements hold true:

(i) The language $L$ is maximal error-detecting for $\delta(m, \ell)$.

(ii) The redundancy of $L$ is asymptotically upper-bounded by $m /(\ell-m) \log _{r} n$, where $n \rightarrow \infty$ and $r$ is the size of the alphabet $X$.

(iii) There exists no language $L^{\prime}$ which is error-detecting for $\delta(m, \ell)$ and whose redundancy is strictly asymptotically upper-bounded by the redundancy of $L$.

(iv) The language $L$ is context-sensitive but not context-free.

The sequence $\left\{h_{k}\right\}_{k \in \mathbb{N}_{0}}$ has the property that $h_{k+1}$ is the length of a shortest word which cannot result in a word of length $h_{k}$ using the maximum number of deletions $D_{m, \ell}\left(h_{k+1}\right)$.

Lemma 5. For all $m, \ell \in \mathbb{N}$ with $\ell>m$, the following statements hold true:

(i) There is a real constant $\beta$ such that for all $k \in \mathbb{N}_{0},(\ell-m) / \ell h_{k+1}+\beta<h_{k}$.

(ii) For the function $g: \mathbb{N}_{0} \rightarrow \mathbb{N}_{0}$ defined by $g(t)=t+1+m+m\lfloor t /(\ell-m)\rfloor$, one has $g(t)=\min \left\{n \in \mathbb{N}_{0} \mid n-D_{m, \ell}(n)>t\right\}$ and $g(t)-D_{m, \ell}(g(t))=t+1$ for all $t \in \mathbb{N}_{0}$, where $D_{m, \ell}(n)$ is the function defined in Lemma 2.

(iii) For all $k \in \mathbb{N}_{0}, h_{k+1}=\min \left\{n \in \mathbb{N}_{0} \mid n-D_{m, \ell}(n)>h_{k}\right\}$.

(iv) For every $n \in \mathbb{N}_{0}$, there is a mapping $f: S \rightarrow X^{n}$, where $S=X^{n-D_{m, \ell}(n)} \cup \cdots \cup X^{n}$, that satisfies the following properties, for all $u, u^{\prime} \in S$ and for $\gamma=\delta(m, \ell):(a)$ $u \in X^{n}$ implies $f(u)=u$; (b) $u \in\langle f(u)\rangle_{\gamma} ;(c)|u|=\left|u^{\prime}\right|$ and $f(u)=f\left(u^{\prime}\right)$ imply $u=u^{\prime} ;$ and $(d)|u|<\left|u^{\prime}\right|$ and $f(u)=f\left(u^{\prime}\right)$ imply $u \in\left\langle u^{\prime}\right\rangle_{\gamma}$.

Proof. For the first statement, we note that $\lfloor x\rfloor \leqslant x$ for all reals $x$ and, therefore, $h_{k+1} \leqslant h_{k}+1+m+m /(\ell-m) h_{k}$. For the second statement, let $t \in \mathbb{N}_{0}$, and let $q$ and $r$ be the unique non-negative integers with $t=q(\ell-m)+r$ and $0 \leqslant r<\ell-$ $m$. For $n=g(t)-1$, one has that $n=q \ell+m+r$ and $D_{m, \ell}(n)=q m+m$. Then, $n>D_{m, \ell}(n)+t$ implies $q m+m>q m+m$ which is a contradiction. On the other hand, for $n=g(t)$, one verifies that $D_{m, \ell}(n)=q m+m$; therefore, $n>D_{m, \ell}(n)+t$ holds true. Hence, $g(t)=\min \left\{n \in \mathbb{N}_{0} \mid n-D_{m, \ell}(n)>t\right\}$. Now, as $g(t+1)$ is the smallest non-negative integer for which $g(t+1)-D_{m, \ell}(g(t+1))>t+1$ and $g$ is strictly monotonically increasing, it follows that $g(t)-D_{m, \ell}(g(t)) \leqslant t+1$. On the other hand, $g(t)-D_{m, \ell}(g(t)) \geqslant t+1$. The third statement follows immediately from the previous one.

For the last statement, we note first that every word $u \in S$ can be written in the form $u_{1} \cdots u_{q} s$, where $q=\lfloor|u| /(\ell-m)\rfloor, u_{i} \in X^{\ell-m}$, and $s \in X^{*}$ with $|s|<\ell-m$. The function $f$ will insert $n-|u|$ symbols in $u$ as follows:

$$
f(u)=a^{m} u_{1} \cdots a^{m} u_{p} a^{t} u_{p+1} \cdots u_{q} s
$$


such that $a \in X, p=\lfloor(n-|u|) / m\rfloor$, and $n-|u|=p m+t$ with $0 \leqslant t<m$. As $\left|a^{m} u_{j}\right|=\ell$ for all $j=1, \ldots, p$, it follows that $u \in\langle f(u)\rangle_{\gamma}$. If $n=|u|$ then $p=t=0$ and $f(u)=u$ as required. The function $f$ is well-defined if we prove $n-|u| \leqslant q m+m$. This is shown as follows: As $|u| \geqslant n-D_{m, \ell}(n)$, the second statement of the lemma implies $g(|u|)>n \Rightarrow 1+m+m q>n-|u|$, as required.

Now consider $u, u^{\prime} \in S$ with $f(u)=f\left(u^{\prime}\right)$. Then,

$$
f(u)=a^{m} u_{1} \cdots a^{m} u_{p} a^{t} u_{p+1} \cdots u_{q} s
$$

and

$$
f\left(u^{\prime}\right)=a^{m} u_{1}^{\prime} \cdots a^{m} u_{p^{\prime}}^{\prime} a^{t^{\prime}} u_{p^{\prime}+1}^{\prime} \cdots u_{q^{\prime}}^{\prime} s^{\prime} .
$$

If $|u|=\left|u^{\prime}\right|$ then $q=q^{\prime}$ and, as $n-|u|=n-\left|u^{\prime}\right|$, it follows that $p=p^{\prime}$ and $t=t^{\prime}$ and, therefore, $u=u^{\prime}$ as required. On the other hand, if $|u|<\left|u^{\prime}\right|$ then $p m+t>p^{\prime} m+t^{\prime}$ which implies that either $p>p^{\prime}$, or $p=p^{\prime}$ and $t>t^{\prime}$. If $p>p^{\prime}$ one has

$$
a^{m} u_{1} \cdots a^{m} u_{p^{\prime}} a^{t^{\prime}}=a^{m} u_{1}^{\prime} \cdots a^{m} u_{p^{\prime}}^{\prime} a^{t^{\prime}}
$$

and

$$
a^{m-t^{\prime}} u_{p^{\prime}+1} a^{m} \cdots a^{m} u_{p} a^{t} u_{p+1} \cdots u_{q} s=u_{p^{\prime}+1}^{\prime} \cdots u_{q^{\prime}}^{\prime} s^{\prime} .
$$

Then, $u_{j}=u_{j}^{\prime}$ for all $j=1, \ldots, p^{\prime}$ and $u^{\prime}=u_{1} \cdots u_{p^{\prime}} a^{m-t^{\prime}} u_{p^{\prime}+1} a^{m} \cdots a^{m} u_{p} a^{t} u_{p+1} \cdots u_{q} s$ which implies $u \in\left\langle u^{\prime}\right\rangle_{\gamma}$ as required. Finally, the case where $p=p^{\prime}$ and $t>t^{\prime}$ can be shown similarly.

Proof of Theorem 5. (i) Suppose $w_{1}, w_{2} \in L$ and $w_{1} \in\left\langle w_{2}\right\rangle_{\gamma}$, where $\gamma=\delta(m, \ell)$. We want to show $w_{1}=w_{2}$. As $\gamma$ permits only deletions, it follows that $\left|w_{1}\right| \leqslant\left|w_{2}\right|$ and that $\left|w_{1}\right|=\left|w_{2}\right|$ implies $w_{1}=w_{2}$. First assume $\left|w_{1}\right|<\left|w_{2}\right|$ and $\left|w_{2}\right|=h_{k}$ for some $k \in \mathbb{N}$. By Lemma $2\left|w_{1}\right| \geqslant h_{k}-D_{m, \ell}\left(h_{k}\right)$ which implies $h_{k-1}<\left|w_{1}\right|<h_{k}$ using Lemma 5(iii). This in turn implies $w_{1} \notin L$ which is a contradiction. Hence, $\left|w_{1}\right|=\left|w_{2}\right|$ and, therefore, $w_{1}=w_{2}$. Thus, $L$ is error-detecting for $\gamma$. Now suppose there is a word $w$ in $X^{*} \backslash L$ such that $L \cup\{w\}$ is error-detecting for $\gamma$. Let $n=|w|$. If $n \leqslant m$ then $\lambda \in\langle w\rangle_{\gamma}$ and, therefore, $w=\lambda$ which is impossible. Hence, there is $k \in \mathbb{N}$ such that $h_{k}<n<h_{k+1}$. By Lemma 5(iii), $n<h_{k+1}$ implies $n-D_{m, \ell}(n) \leqslant h_{k}$. By Lemma 2, there is $z \in X^{h_{k}}$ with $z \in\langle w\rangle_{\gamma}$. But, as $L \cup\{w\}$ is error-detecting for $\gamma, w=z \in L$; a contradiction. Hence, $L$ is maximal error-detecting for $\gamma$.

(ii) Assume that $L=\left\{w_{i} \mid i \in \mathbb{N}_{0}\right\}$ such that $\left|w_{i}\right| \leqslant\left|w_{i+1}\right|$ for all $i \in \mathbb{N}_{0}$. Let $n \in \mathbb{N}_{0}$ and let $s_{n}=\left|w_{n}\right|-1$. Then $N_{L}\left(s_{n}\right) \leqslant n$ and there is $k \in \mathbb{N}_{0}$ such that $h_{k} \leqslant s_{n}<h_{k+1}$. Moreover, $N_{L}\left(s_{n}\right)=\sum_{i=0}^{k} r^{h_{i}}$. As $r^{h_{k}} \leqslant N_{L}\left(s_{n}\right)$, one has $h_{k} \leqslant \log _{r} n$. Then, using Lemma 5(i) and $s_{n}<h_{k+1}$, it follows that $(\ell-m) / \ell s_{n}+\beta<\log _{r} n$ and, therefore, the redundancy of $L$ is asymptotically upper-bounded by $\ell /(\ell-m) \log _{r} n-\log _{r} n=m /(\ell-m) \log _{r} n$.

(iii) Assume that there is a language $L^{\prime}$ whose redundancy is strictly asymptotically upper-bounded by the redundancy of $L$. Then, the set $\left\{n \in \mathbb{N}_{0} \mid N_{L^{\prime}}(n) \leqslant N_{L}(n)\right\}$ is finite by Lemma 3 . We obtain a contradiction by showing that $N_{L^{\prime}}\left(h_{k}\right) \leqslant N_{L}\left(h_{k}\right)$ for all 
$k \in \mathbb{N}_{0}$. For each $k \in \mathbb{N}_{0}$, let $M_{k}=L^{\prime} \cap S$ with $M_{0}=L^{\prime} \cap X^{0}$, where $S=X^{1+h_{k-1}} \cup \cdots \cup$ $X^{h_{k}}$. By the definition of $h_{k}$ and Lemma 5(ii), one has $h_{k}=g\left(h_{k-1}\right)$ and $h_{k}-D_{m, \ell}\left(h_{k}\right)=$ $1+h_{k-1}$. Hence, there is a function $f: S \rightarrow X^{h_{k}}$ as defined in Lemma 5(iv). Let $f_{k}: M_{k} \rightarrow X^{h_{k}}$ be the restriction of $f$ on $M_{k}$. We show that $f_{k}$ is injective. Let $u, u^{\prime} \in M_{k}$ with $f_{k}(u)=f_{k}\left(u^{\prime}\right)$ and assume $|u| \leqslant\left|u^{\prime}\right|$. If $|u|=\left|u^{\prime}\right|$ then $u=u^{\prime}$ as required. If $|u|<\left|u^{\prime}\right|$ then $u \in\left\langle u^{\prime}\right\rangle_{\gamma}$ which is a contradiction as $L^{\prime}$ is error-detecting for $\gamma=\delta(m, \ell)$. Hence, $f_{k}$ is injective and this implies $\left|M_{k}\right|=\left|f_{k}\left(M_{k}\right)\right| \leqslant|X|^{h_{k}}$ for all $k \in \mathbb{N}_{0}$. Then, one has

$$
N_{L^{\prime}}\left(h_{k}\right)=\left|M_{0} \cup \cdots \cup M_{k}\right| \leqslant \sum_{i=0}^{k}|X|^{h_{i}}=N_{L}\left(h_{k}\right),
$$

as required.

(iv) For any $k$ in $\mathbb{N}_{0}$, one has $h_{k+1}>h_{k}+1+m h_{k} /(\ell-m)$ which implies that $h_{k}$ grows exponentially with respect to $k$. But, as $\left\{h_{k} \mid k \in \mathbb{N}_{0}\right\}$ is the length set of $L$, the language $L$ cannot be context-free.

Now it is well-known that a language is context-sensitive if and only if there is a linearly bounded Turing machine that accepts exactly the words of the language (see [15], for instance). In the case of the language $L$, given an input word $w$ between the special markers $\$$ and \#, a linearly bounded machine needs to test whether $|w| \in\left\{h_{k} \mid k \in \mathbb{N}_{0}\right\}$ using only the space between the special markers. This can be done incrementally by testing first whether $|w|=h_{0}$ and then, in general, if $|w| \neq h_{k}$ test whether $|w|=h_{k+1}$.

\section{Discussion}

In this paper, we have presented some initial results on error-detection at the general level of $P$ - and SID-channels, and examined certain error-detecting capabilities of uniform, solid, and shuffle codes. Some potentially interesting questions that arise from this work are the following:

(1) With Theorem 1(ii) in mind, what other bounds exist on the insertion/deletiondetecting capabilities of languages?

(2) Is it possible to show that solid codes possess stronger error-detecting capabilities than the one shown in Theorem 4 for the SID-channel $\sigma \odot \imath \odot \delta(1, \ell)$ ?

(3) How large is the intersection between certain shuffle codes and solid codes? In view of Theorem 4 and Proposition 3, it appears that codes in that intersection provide certain $*$-error-detecting capabilities for free.

(4) Are there examples of variable-length codes which are $*$-error-detecting for $\delta(m, \ell)$ with delay 0 and more efficient than the uniform code shown in Section 5 ?

(5) What is the asymptotic redundancy of the language $L$ of Section 5? The answer is interesting as $L$ is most efficient with the property of detecting errors of the deletion type. 


\section{References}

[1] P. Beckmann, The Structure of Language: A New Approach, The Golem Press, Boulder, CO, 1972.

[2] J. Berstel, D. Perrin, Theory of Codes, Academic Press, Orlando, FL, 1985.

[3] V. Bruyère, Maximal codes with bounded deciphering delay, Theoret. Comput. Sci. 84 (1991) 53-76.

[4] R.M. Capocelli, L. Gargano, U. Vaccaro, Decoders with Initial State Invariance for Multivalued Encodings, Theoret. Comput. Sci. 86 (1991) 365-375.

[5] J. Duske, H. Jürgensen, Codierungstheorie, BI Wissenschaftsverlag, Mannheim, 1977.

[6] W.E. Hartnett (Ed.), Foundations of Coding Theory, Reidel, Boston, 1974.

[7] H. Jürgensen, M. Katsura, S. Konstantinidis, Maximal solid codes, J. Automata Languages Combin. 6 (2001) 25-50.

[8] H. Jürgensen, S. Konstantinidis, Error correction for channels with substitutions, insertions, and deletions, in: J.-Y. Chouinard, P. Fortier, T.A. Gulliver (Eds.), Information Theory and Applications 2, Fourth Canadian Workshop on Information Theory, Lecture Notes in Computer Science, Vol. 1133, Springer, Berlin, 1996, pp. 149-163.

[9] H. Jürgensen, S. Konstantinidis, Codes, in: G. Rozenberg, A. Salomaa (Eds.), Handbook of Formal Languages, Vol. I, Springer, Berlin, 1997, pp. 511-607.

[10] S. Konstantinidis, An algebra of discrete channels that involve combinations of three basic error types, Inform. and Comput. 167 (2001) 120-131.

[11] N.H. Lâm, Finite maximal solid codes, Preprint 98/17, Vietnam National Centre for Natural Science and Technology, Institute of Mathematics, 1998, Theoret. Comput. Sci. 262 (2001) 333-347.

[12] V.I. Levenshtein, Self-adaptive automata for decoding messages, Dokl. Akad. Nauk. SSSR 141 (1961) 1320-1323 (English translation: Soviet Phys. Dokl. 6 (1962) 1042-1045).

[13] V.I. Levenshtein, Binary codes capable of correcting deletions, insertions, and reversals, Dokl. Akad. Nauk. SSSR 163 (1965) 845-848 (in Russian) (English translation: Soviet Phys. Dokl. 10 (1966) 707-710).

[14] V.I. Levenshtein, On the redundancy and delay of decodable coding of natural numbers, Problemy Kibernet. 20 (1968) 173-179 (in Russian) (English translation: Systems Theory Res. 20 (1971) 149-155).

[15] A. Mateescu, A. Salomaa, Aspects of classical language theory, in: G. Rozenberg, A. Salomaa (Eds.), Handbook of Formal Languages, Vol. I, Springer, Berlin, 1997, pp. 175-251.

[16] S. Roman, Coding and Information Theory, Springer, New York, 1992.

[17] G. Rozenberg, A. Salomaa (Eds.), Handbook of Formal Languages, Vol. I, Springer, Berlin, 1997.

[18] H.J. Shyr, Free Monoids and Languages, 2nd ed., Hon Min Book Company, Taichung, 1991.

[19] S. Yu, Regular languages, in: G. Rozenberg, A. Salomaa (Eds.), Handbook of Formal Languages, Vol. I, Springer, Berlin, 1997, pp. 41-110. 